\title{
Erratum: Phenomenology of QCD threshold resummation for gluino pair production at NNLL
}

\author{
Torsten Pfoh \\ Deutsches Elektronensynchrotron DESY, \\ Platanenallee 6, D-15738 Zeuthen, Germany \\ E-mail: torsten.pfoh@desy.de
}

ERRATUm to: JHEP05(2013)044

ARXiv EPRINT: 1302.7202 


\begin{tabular}{|cccc|}
\hline$m_{\tilde{g}}[\mathrm{GeV}]$ & $\sqrt{s}=7 \mathrm{TeV}$ & $8 \mathrm{TeV}$ & $14 \mathrm{TeV}$ \\
\hline & $\sigma^{\text {res,NNLO }}$ approx $[\mathrm{pb}]$ & for MSTW 2008 NNLO \\
\hline 800 & $0.0351\left(\begin{array}{l}0.0407 \\
0.0306\end{array}\right)$ & $0.0828\left(\begin{array}{l}0.0948 \\
0.0729\end{array}\right)$ & $1.4636\left(\begin{array}{l}1.6218 \\
1.3321\end{array}\right)$ \\
\hline 1000 & $0.0037\left(\begin{array}{l}0.0044 \\
0.0032\end{array}\right)$ & $0.0104\left(\begin{array}{l}0.0121 \\
0.0090\end{array}\right)$ & $0.2952\left(\begin{array}{l}0.3294 \\
0.2665\end{array}\right)$ \\
\hline \multicolumn{5}{|c|}{$\sigma^{\text {res,NNLO }}$ approx $[\mathrm{pb}]$ for ABM11 NNLO } \\
\hline 800 & $0.0142\left(\begin{array}{l}0.0166 \\
0.0125\end{array}\right)$ & $0.0361\left(\begin{array}{l}0.0417 \\
0.0320\end{array}\right)$ & $0.8489\left(\begin{array}{l}0.9459 \\
0.7722\end{array}\right)$ \\
\hline 1000 & $0.0014\left(\begin{array}{l}0.0016 \\
0.0012\end{array}\right)$ & $0.0041\left(\begin{array}{l}0.0048 \\
0.0036\end{array}\right)$ & $0.1557\left(\begin{array}{l}0.1750 \\
0.1408\end{array}\right)$ \\
\hline
\end{tabular}

Table 1. Hadronic cross section at $\sqrt{s}=7,8$, and $14 \mathrm{TeV}$, evaluated by using MSTW 2008 and ABM11 PDF sets. The ratio $m_{\widetilde{q}} / m_{\widetilde{g}}$ is kept fixed to the value $4 / 5$. The numbers in brackets correspond to the hadronic cross section evaluated at $\mu=m_{\tilde{g}} / 2$ (upper number) and $\mu=2 m_{\tilde{g}}$. PDF errors are not included.

There has been an error in my FORTRAN code that computes the resummed cross section. This has in part been related to the shifted argument $N+1$ in the resummation formula (2.2). Moreover, within the Mellin inversion given by eq. (4.2), Mellin- $N$ should be identified with $c(z)$, not with $c(z)-1$ as stated in the article. The numerical consequences are as follows:

At the partonic level, there is a better agreement between the the NNLO threshold limit and the expansion in $\alpha_{s}$ of the resummation formula. The NNLL resummed cross section merges with the NNLO approximation away from the threshold, see corrected figure 1.

The error bars due to scale variation are now rather symmetric with respect to the central values, see corrected figure 2 .

After convolution with the PDFs, the NNLL resummation gives rise to a small enhancement compared to the fixed next-to-leading-order cross section. The K-factor $K_{\mathrm{NNLL}}$ is about $1.04-1.09$, depending on the gluino mass, the center of mass energy, the chosen PDF set and the related value of $\alpha_{s}\left(M_{Z}\right)$.

The explicit numbers in the last column of table 1 need to be corrected according to the table 1.

Finally, at the hadronic level, the error of the resummed result due to scale variation has shrunk and no longer exceeds the uncertainty of the fixed NLO computation, see corrected figure 3 . 

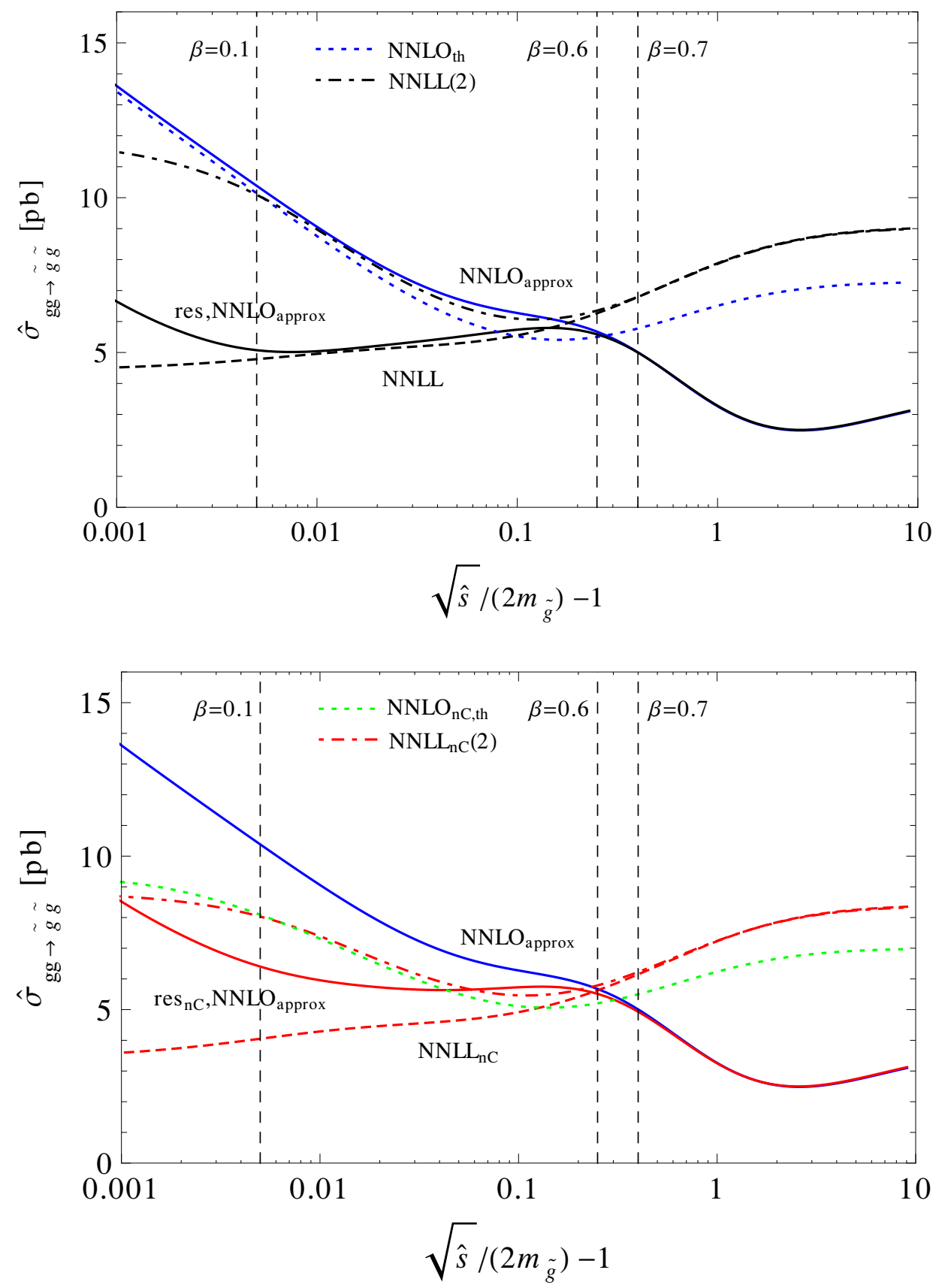

Figure 1. Partonic cross section for gluon fusion versus the energy above the production threshold normalized to the gluino pair mass. The renormalization and factorization scales have been set to $\mu=m_{\widetilde{g}}=800 \mathrm{GeV}$. We plot the NNLO approximation which is exact up to NLO, and the NNLO threshold limit $\mathrm{NNLO}_{\text {th }}$ which contains only threshold-enhanced contributions and NLO constants. Moreover, we show the NNLL resummed cross section and its expansion in $\alpha_{s}$ up to second order, NNLL(2), as well as the resummed partonic cross section matched onto the NNLO approximation. First panel: coulomb corrections are included at fixed order in the resummation formula. Second panel: coulomb corrections are neglected during resummation but kept in the NNLO approximation. 


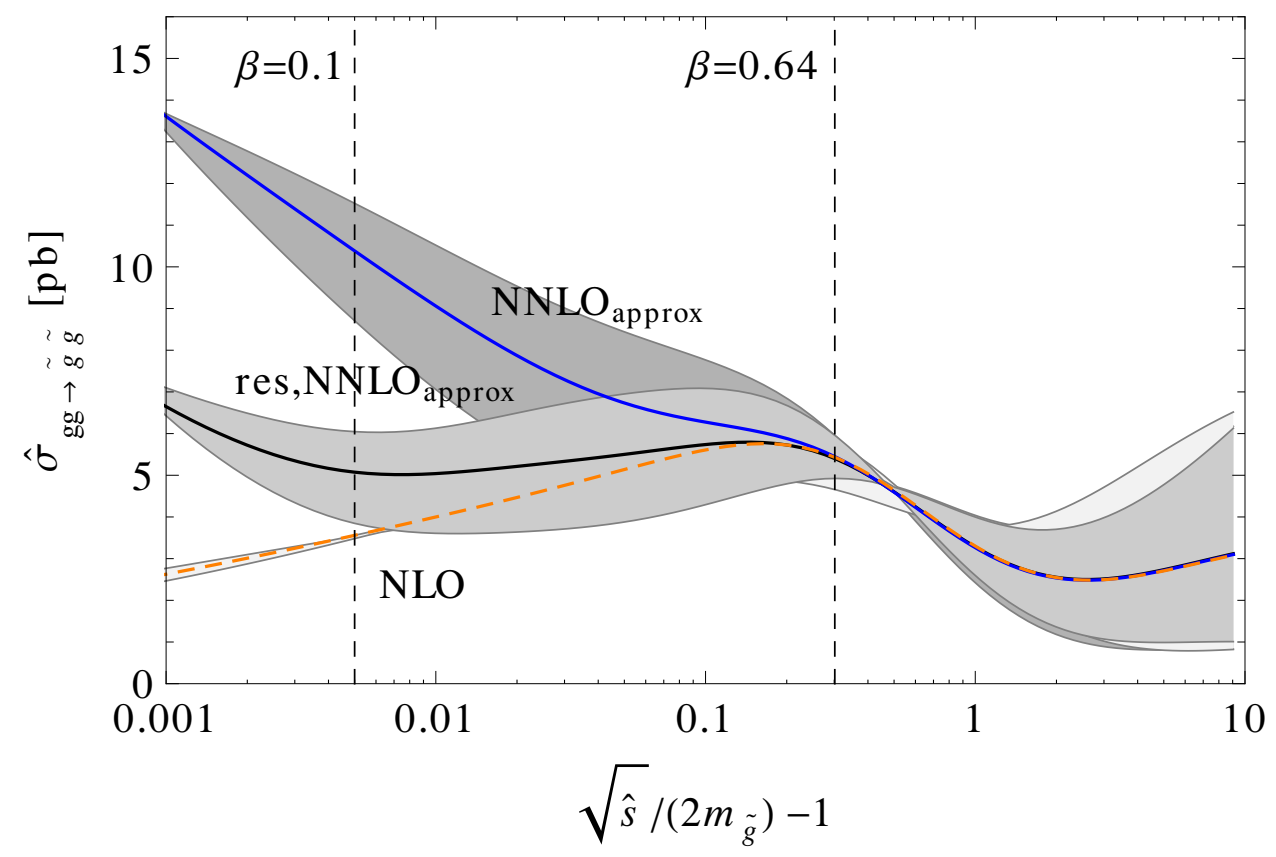

Figure 2. Comparison of the resummed partonic cross section to the exact NLO result and the NNLO approximation. The scale is varied within the interval $\mu \in\left[m_{\tilde{g}} / 2,2 m_{\tilde{g}}\right]$ around $\mu=m_{\widetilde{g}}=$ $800 \mathrm{GeV}$.

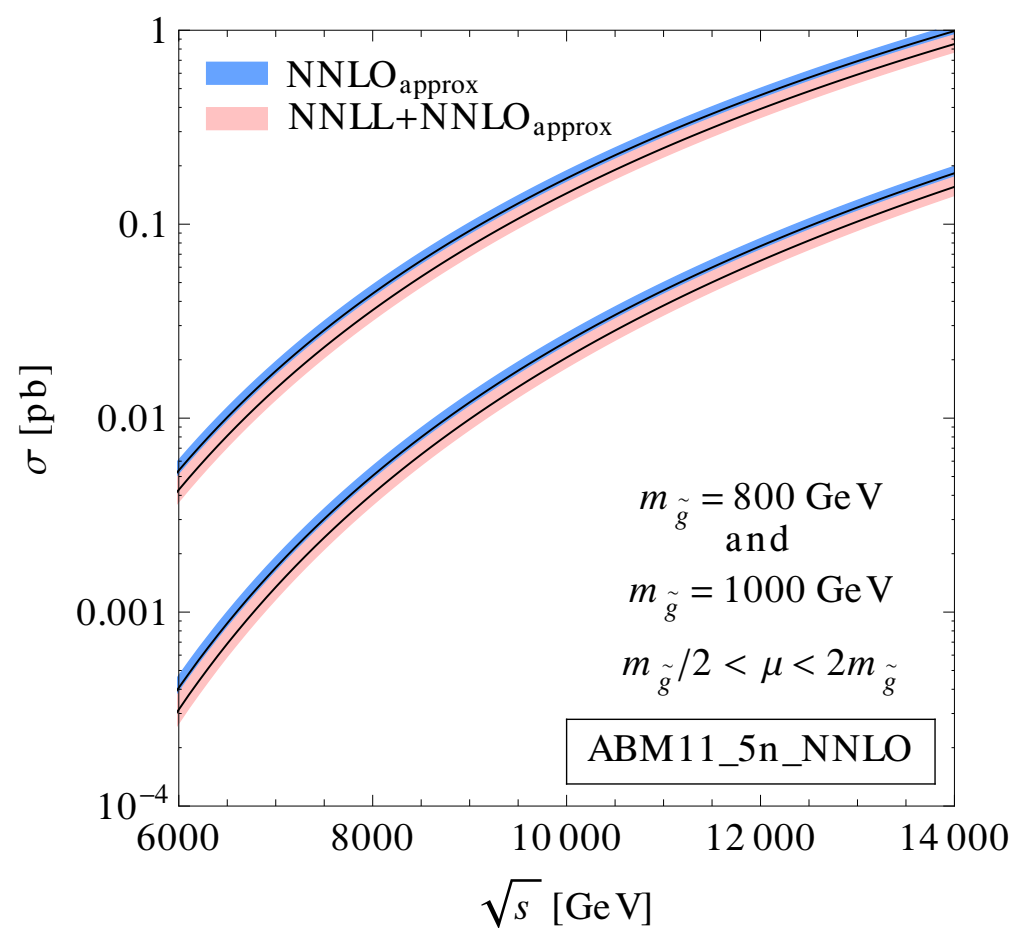

Figure 3. Inclusive hadronic cross section versus the squared cms energy. The error bars refer to scale variation only. 